\title{
Non-thermal radio emission from Wolf-Rayet stars
}

\author{
Jessica M. Chapman ${ }^{1,2}$, Claus Leitherer ${ }^{3}$, and Bärbel Koribalski ${ }^{2}$ \\ ${ }^{1}$ Anglo-Australian Observatory, \\ PO Box 296, Epping, NSW 2121, Australia \\ ${ }^{2}$ Australia Telescope National Facility, \\ PO Box 76, Epping, NSW 2121, Australia \\ ${ }^{3}$ Space Telescope Science Institute, \\ 3700 San Martin Drive, Baltimore, MD 21218, USA
}

We have used the Australia Telescope Compact Array to search for radio continuum emission at $\lambda 3,6,13$ and $20 \mathrm{~cm}$ from a distance-limited sample of 36 southern Wolf-Rayet stars within $3 \mathrm{kpc}$ of the Sun. The observations were taken between 1994 and 1997. Radio continuum emission has been detected from 16 sources at $3 \mathrm{~cm}$. Of these 11 sources were detected at $6 \mathrm{~cm}$, seven sources were detected at $13 \mathrm{~cm}$ and 2 sources were detected at $20 \mathrm{~cm}$ (Chapman et al. 1999).

From the ATCA data we have identified six WR stars, WR 14, WR 39, WR 48, WR 90, WR 105 and WR 112 which have non-thermal radio continuum emission. From the detection rate we estimate that at least $40 \%$ of the WR stars have non-thermal emission at $\mathrm{cm}$ wavelengths. Table 1 lists the six nonthermal sources. Columns 1 and 2 gives the source identifications and spectral types. Column 3 gives the best available distances. Columns 4 and 5 give the radio spectral indices and the wavelength ranges for which the indices have been calculated. Column 6 gives their $5 \mathrm{GHz}$ radio luminosities.

Table 1. Wolf Rayet stars with confirmed non-thermal radio emission

\begin{tabular}{rcrrll}
\hline WR & spectral type & $\begin{array}{c}d \\
(\mathrm{kpc})\end{array}$ & $\alpha$ & $\begin{array}{c}\lambda \\
(\mathrm{cm})\end{array}$ & $\begin{array}{c}L_{5 \mathrm{GHz}} \\
\left(10^{19} \mathrm{erg} \mathrm{s}^{-1} \mathrm{~Hz}^{-1}\right)\end{array}$ \\
\hline & & & & \\
14 & WC7 & 2.9 & -1.0 & $3-6$ & 0.5 \\
39 & WC7+WC7 & 5.7 & 0.0 & $3-20$ & 3.4 \\
48 & WC6+O9.5 & 2.4 & -0.4 & $3-13$ & 1.4 \\
90 & WC7 & 2.0 & 0.0 & $3-6$ & 0.5 \\
105 & WN8 & 1.6 & -0.3 & $3-6$ & 1.4 \\
112 & WC9 & 1.2 & $<0$ & $3-20$ & $<0.1$ \\
\hline
\end{tabular}

In all cases the non-thermal radio emission almost certainly originates from an interaction between the WR stellar wind with the wind from a massive companion star. The radio luminosities and spectral indices are in good agreement with theoretical models where synchroton emission is generated from relativistic electrons which are accelerated in strong shocks (Eichler \& Usov 1993). For WR 90, WR 105 and WR 112 no binary companions have been identified. However the 


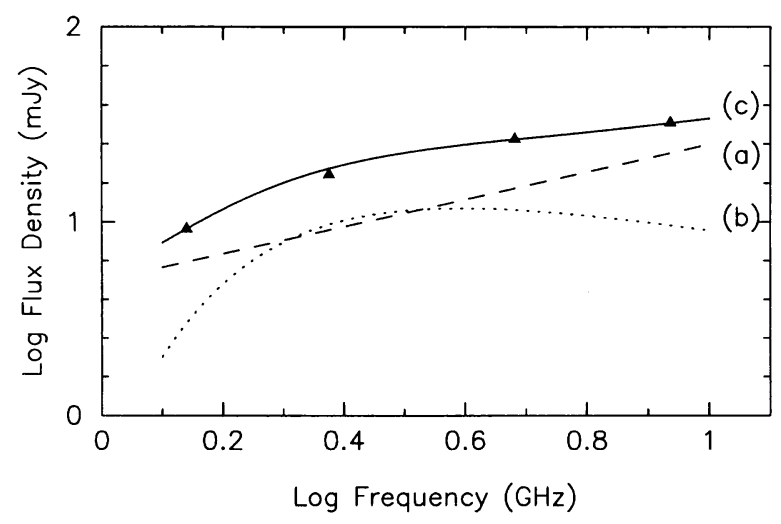

Figure 1. Model spectra for WR $11\left(\gamma^{2}\right.$ Velorum, WC8+O9I $)$

radio emission is variable on a timescale of years indicating that orbital motions are likely. The radio variability is likely to be due to a variable amount of absorption through the ionized winds of the WR stars. WR 14 may also have a binary companion, indicated by variability in optical polarisation data (Drissen et al. 1992).

WR 39 is of particular interest as it is the first known example of a possible wind-wind interaction occuring between two WR stars. We propose that the non-thermal emission occurs from an interaction with the wind of the WR star WR38B which is offset by approximately $3^{\prime \prime}$. The small angular separation between the two stars and similarities in their optical spectra show that they are likely to have been formed as a pair (Shara et al. 1991).

Another source of interest is WR $11\left(\gamma^{2}\right.$ Velorum) which has an O9 companion star with an orbital period of 78.53 days. The spectral index of WR 11 decreases with frequency between 1.4 and $8.6 \mathrm{GHz}$. This is interpreted as evidence for a strongly attenuated non-thermal component to the observed emission. This is illustrated by the model shown in Fig. 1. The dashed line (a) shows the thermal emission from an ionized wind with a spectral index of +0.7 . The dotted line (b) shows non-thermal emission with a spectral index of -0.5 which is attenuated by free-free absorption. The solid line shows the sum of the two components. The triangles show the measured ATCA flux densities which were used to determine the model parameters.

\section{References}

Chapman, J.M., Leitherer, C., Koribalski, B., Bouter, R., Storey, M. 1999 ApJ in press

Drissen, L., Robert, C., Moffat, A.F.J. 1992, ApJ 386, 288

Eichler, D., Usov, V. 1993, ApJ 402, 271

Shara, M.M., Moffat, A.F.J., Smith, L.F., Potter, M. 1991, AJ 102, 716 